\title{
CAPACIDAD DIAGNÓSTICA DE LAS ESCALAS BRADEN Q Y NORTON PARA ÚLCERAS POR PRESIÓN EN PACIENTES PEDIÁTRICOS DE UNIDADES CRÍTICAS
}

\author{
DIAGNOSTIC CAPACITY OF THE BRADEN Q AND NORTON SCALES FOR PRESSURE \\ ULCERS IN PEDIATRIC PATIENTS OF CRITICAL UNITS
}

CAPACIDADE DIAGNÓSTICA DAS ESCALAS BRADEN Q E NORTON PARA ULCERES PRESSORES EM PACIENTES PEDIÁTRICOS DE UNIDADES CRÍTICAS

\section{Jenny Caniupán}

Universidad Autónoma de Chile. Temuco, Chile.

ORCID: 0000-0002-5168-3572

\section{Edith Rivas}

Universidad de La Frontera. Temuco, Chile.

edith.rivas@ufrontera.cl

ORCID: 0000-9832-4534

\section{Luis Bustos \\ Universidad Frontera. Temuco, Chile. \\ ORCID: 0000-0002-6917-3239}

DOI: https://doi.org/10.22235/ech.v7i1.1539

Recibido: 28/09/2017

Aceptado: 08/03/2018

Resumen: El objetivo de la investigación fue conocer la capacidad diagnóstica de las escalas Braden Q y Norton para el desarrollo de úlceras por presión en pacientes pediátricos de Unidades Críticas en un hospital de alta complejidad en Chile. Es un estudio transversal de comparación de pruebas diagnósticas en pacientes ingresados a unidades críticas. La muestra fue no probabilística, por conveniencia de 118 niños. Se analizaron ambas escalas respetando los preceptos, para comparar la capacidad discriminativa de dos pruebas diagnósticas y verificar que ambas sean medidos simultáneamente aplicadas sobre los mismos sujetos. La investigación contó con aprobación de comité de Ética Servicio de Salud y Consentimiento Informado firmado de los padres de los niños. Se determinó que la sensibilidad de la escala Braden Q, a las 24 y 48 horas, es menor que la presentada por la Norton en las mismas evaluaciones; presenta valor predictivo positivo más alto en ambas mediciones, como también la razón de probabilidad $(+)$. Se concluye que Braden Q es la escala idónea para valorar el riesgo de desarrollar UPP en la población pediátrica; presenta mayor confiabilidad.

Palabras clave: Escalas, Úlcera por Presión, Unidades de Cuidados Intensivos Pediátricos. Enfermeras Pediátricas. 


\begin{abstract}
The objective of the investigation was to evaluate the diagnostic capacity of the Braden Q and Norton scales for the development of pressure ulcers in pediatric patients of Critical Units in a highly complex hospital in Chile. It is a cross-sectional study comparing diagnostic tests in patients admitted to critical units. The sample was non-probabilistic, for the convenience of 118 children. Both scales were analyzed respecting the precepts, in order to compare the discriminative capacity of two diagnostic tests verifying that both are measured simultaneously while applied on the same subjects. The research was approved by the Ethics Committee, Health Service and Informed Consent signed by the parents of the children. It was determined that the sensitivity of the Braden Q scale, at 24 and 48 hours, is lower than Norton's in the same evaluations; it has a higher positive predictive value in both measurements, as well as the probability ratio $(+)$. It is concluded that Braden $Q$ is the ideal scale to assess the risk of developing pressure ulcers in the pediatric population; it presents greater reliability.
\end{abstract}

Keywords: Scales, Pressure Ulcer, Pediatric Intensive Care Units. Pediatric nurses.

Resumo: O objetivo da investigação foi conhecer a capacidade diagnóstica das escalas Braden Q e Norton, para o desenvolvimento de úlceras de pressão (UPP) em pacientes pediátricos de unidades críticas, em um hospital altamente complexo, no Chile. É um estudo transversal que compara testes diagnósticos em pacientes admitidos em unidades críticas. A amostra não era probabilística, para a conveniência de 118 crianças. Ambas as escalas foram analisadas respeitando os preceitos, para comparar a capacidade discriminativa de dois testes de diagnóstico e verificar que ambos são medidos simultaneamente, aplicados nos mesmos assuntos. A pesquisa foi aprovada pelo Comitê de Ética, Serviço de Saúde e Consentimento Informado assinado por pais de crianças. Determinou-se que a sensibilidade da escala de Braden Q, às 24 e 48 horas, é menor que a apResentada por Norton nas mesmas avaliações, possui maior valor preditivo positivo em ambas as medidas, bem como a razão de probabilidade $(+)$. Conclui-se que Braden Q é a escala ideal para avaliar o risco de desenvolver UPP na população pediátrica, e apresenta maior confiabilidade.

Palavras-chave: Escalas, Lesão por Pressão, Unidades de Terapia Intensiva Pediátrica, Enfermeiras Pediátricas.

\title{
INTRODUCCIÓN
}

Los niños hospitalizados en unidades críticas presentan mayor susceptibilidad de presentar zonas de presión, con desarrollo de úlceras por presión (UPP) debido a que están sometidos especialmente a inmovilidad prolongada, alteración del estado de conciencia, disminución de sensibilidad y largas estadías (1). Las UPP son lesiones de rápida aparición, lenta y compleja curación, que representan un problema biomédico relevante para la salud; corresponden a una medición de la calidad de atención en salud y su aparición representa una grave lesión iatrogénica a nivel hospitalario.

La evidencia sobre evaluación de riesgo de úlceras por presión en la población pediátrica es limitada y en Chile no existen estudios de esta naturaleza. Se señala que el 95\% de las UPP en población total se pueden prevenir (2) y que en unidades críticas se 
presenta mayor riesgo de desarrollarlas, ya que si bien la incidencia en la población pediátrica es de $3.36 \%$ (3), en las unidades de cuidados intensivos pediátricos este porcentaje se ve ampliamente superado con una incidencia de $29 \%$ (4), por lo que su prevención y tratamiento deben ser prioridad.

La identificación de pacientes susceptibles de desarrollo de UPP resulta fundamental puesto que de ser pesquisados con la escala adecuada se podrán instaurar precozmente los cuidados focalizados en evitar su aparición (5).

La evaluación de riesgo de úlceras por presión no ha sido considerada una práctica habitual en menores (6) y cuando se realiza, se han utilizado instrumentos diseñados para población adulta.

En la Unidad de estudio se aplica diariamente la escala Norton, escala diseñada y validada para ser utilizada en la población adulta (7) por normativa de calidad vigente en el hospital (8). Esta escala se utiliza para categorizar el riesgo de desarrollo de UPP tanto en pacientes adultos como pediátricos, no cumpliendo con el objetivo de prácticas de prevención recomendadas para la población pediátrica, ya que la escala Braden Q (9) es el instrumento ampliamente utilizado, validado y fiable para uso en este grupo $(10,11)$.

El objetivo de este estudio fue determinar la capacidad diagnóstica de las escalas Braden Q y Norton para el desarrollo de úlceras por presión en pacientes pediátricos de Unidades Críticas, en un hospital de alta complejidad del sur de Chile durante los años 2014-2015.

\section{METODOLOGÍA}

Estudio transversal de comparación de pruebas diagnósticas (12), donde el gold standard es la escala Braden Q. Se realizaron tres mediciones de riesgo de desarrollar UPP con las escalas Norton y Braden Q, a las 0 horas (primeras 12 horas de ingreso) 24 y 48 horas de hospitalización. La muestra fue no probabilística, por conveniencia de 118 niños hospitalizados en las Unidades Críticas Pediátricas. Los criterios de selección fueron: niños mayores de 30 días y menores de 14 años, 11 meses y 29 días, hospitalizados en Unidades críticas, cuyos padres acepten la participación de sus hijos en el estudio mediante la firma de Formulario de Consentimiento Informado.

Se analizaron ambas escalas respetando los preceptos. Para comparar la capacidad discriminativa de dos pruebas diagnósticas es importante verificar que ambas deben ser medidas simultáneamente, aplicadas sobre los mismos sujetos y contrastadas.

Para el análisis de los datos se utilizó el programa STATA 11.0v. La investigación contó con la aprobación del Comité de Ética del Servicio de Salud (Anexos), firma de Formulario de Consentimiento Informado por parte de los padres de niños participantes. Además, se consideraron aspectos éticos, según los principios de Ezequiel Emanuel (13).

\section{RESULTADOS}

La muestra fue de 118 pacientes, 55 de ellos (46\%) hospitalizados en Unidad de Cuidados Intensivos (UCIP). El diagnóstico de mayor prevalencia fue de tipo respiratorio $(52 \%)$. No se registraron pacientes con desarrollo de úlceras previas al ingreso; 76 niños (64\%) no tenían días previos de hospitalización. La edad promedio de 
la población es de 45,36 meses ( $(53.14 \mathrm{DS})$, con mínimo de 1 mes y máximo de 178 meses. De los pacientes ingresados a UCIP el 16\% ingresó conectado a ventilación mecánica, lo cual disminuyó a 14\% a las 48 horas de hospitalización. El 20\% y 14\% de la muestra estuvo con sedación y paralización, lo cual disminuyó a las 48 horas a $15 \%$ y $11 \%$, respectivamente. Las medidas de prevención de UPP para estos pacientes fueron: uso de colchón antiescaras $54 \%$ a las 0 horas, disminuyendo a $51 \%$ a las 48 horas. Lubricación de la piel: al 23\% se realizó cada dos horas, el 6\% se programó cada 3 horas y el $26 \%$ quedó con lubricación de la piel cada 4 horas, medidas sin mucha variación en las posteriores evaluaciones. Cambio de posición: al 33\% se les programó cada 2 horas, $6 \%$ cada 3 horas y 44\% de los niños fueron cambiados de posición cada 4 horas, como se muestra en la tabla 1.

Presencia de UPP en la evaluación realizada a las 24 horas, según la escala Braden Q: 7 niños presentaron UPP, lo que aumentó a 12 pacientes a las 48 horas; con Norton, fueron 10 y 16 respectivamente, si bien la sensibilidad de la escala Braden Q a las 24 y 48 horas es menor que la presentada por Norton en las mismas evaluaciones; su valor predictivo positivo en ambas mediciones es más alto, como también la razón de probabilidad positiva que presenta esta escala. Tabla $\mathrm{N}^{\circ} 2$.

Tabla $N^{\circ} 1$ : Medidas de prevención de úlcera por presión, niños en Unidades Críticas.

\begin{tabular}{l|c|c|c}
\hline & $\begin{array}{c}0 \mathrm{hrs} \\
\left(\mathrm{n}^{*}=118\right)\end{array}$ & $24 \mathrm{hrs}\left(\mathrm{n}^{*}=114\right)$ & $\begin{array}{c}48 \mathrm{hrs} \\
(\mathrm{n}+=97)\end{array}$ \\
\hline Ventilación mecánica & 16,9 & 15,8 & 14,4 \\
\hline Sedación & 20,3 & 19,3 & 15,4 \\
\hline Paralización & 14,4 & 15,8 & 11,3 \\
\hline Uso colchón antiescara & 54,2 & 50,9 & 51,6 \\
\hline Lubricación de piel c/2 hrs. & 23,7 & 25,4 & 28,9 \\
\hline Lubricación de piel c/3 hrs. & 6,8 & 5,3 & 3,1 \\
\hline $\begin{array}{l}\text { Lubricación de la piel c/ 4 } \\
\text { hrs. }\end{array}$ & 26,3 & 23,7 & 26,8 \\
\hline Cambio de posición c/2 hrs. & 34 & 30 & 30 \\
\hline Cambio de posición c/3hrs. & 7 & 7 & 5,2 \\
\hline $\begin{array}{l}\text { Cambio de posición c/ } 4 \\
\text { hrs. }\end{array}$ & 44,1 & 47,4 & 46,4 \\
\hline
\end{tabular}

valores expresado en \%

Fuente: Elaboración propia (2017) 
Tabla N²: Presencia de escara en niños críticos, según escala Braden Q y Norton

\begin{tabular}{|c|c|c|c|c|c|c|c|c|c|}
\hline \multicolumn{4}{|c|}{ Presencia de éscara } & & & & & & \\
\hline & & $\mathrm{Si}$ & No & & & & & & \\
\hline \multicolumn{4}{|c|}{ Braden Q } & SN* & SP* & VP+* & VP- & LR+ & LR- \\
\hline \multirow[t]{2}{*}{ 24h } & $\mathrm{Si}$ & 7 & 19 & 53,8 & 81,2 & 26,9 & 93,2 & 2,8 & 0,5 \\
\hline & No & 6 & 82 & & & & & & \\
\hline \multirow[t]{2}{*}{$48 \mathrm{~h}$} & Si & 12 & 11 & 60,0 & 85,7 & 52,2 & 89,2 & 4,2 & 0,4 \\
\hline & No & 8 & 66 & & & & & & \\
\hline \multicolumn{10}{|c|}{ Norton } \\
\hline \multirow[t]{2}{*}{24} & Si & 10 & 55 & 76,9 & 45,5 & 15,4 & 93,9 & 1,41 & 0,5 \\
\hline & No & 3 & 46 & & & & & & \\
\hline \multirow[t]{2}{*}{48} & $\mathrm{Si}$ & 16 & 33 & 80,0 & 57,1 & 32,7 & 91,7 & 1,8 & 0,3 \\
\hline & No & 4 & 44 & & & & & & \\
\hline
\end{tabular}

*n, total de niños ingresados al estudio; valores expresado en \%

*Sensibilidad; **especificidad; ${ }^{* *}$ valor predictivo positivo; valor predictivo negativo; ${ }^{\circ}$ Likelihood ratio positivo; ${ }^{\circ 00}$ likelihood negativo

Fuente: Elaboración propia (2017)

\section{DISCUSIÓN}

Braden Q si bien muestra una sensibilidad menor en relación a Norton (escala diseñada para adultos), su especificidad en todas las mediciones es mayor. El mayor valor predictivo positivo y razón de probabilidad $(+)$ de Braden Q permiten recomendarla para ser utilizada en la población pediátrica, tal como se expuso en el año 2003 (14) en un estudio de prevalencia donde se evidencia que esta escala es un instrumento válido y fiable para ser utilizado en este grupo etario. Esto resulta de interés y le otorga valor a 
los resultados obtenidos, ya que se evidencia que las escalas deben ser utilizadas para el grupo para el que fueron diseñadas (15). Además la población pediátrica presenta zonas propias y específicas de aparición de las UPP (16) las cuales requieren de instrumentos válidos y confiables para su detección (17) temprana y/o prevención de las mismas (15). Esto anula el uso de la escala de Norton por su baja efectividad en la población pediátrica, obteniendo un resultado sobrevalorado para este grupo etario, según los resultados obtenidos.

Resulta importante asimismo destacar que la población de estudio presenta un mínimo de edad de 1 mes y máximo de 178 meses; por ende, el grupo de estudio está dentro del rango de edad para el cual se recomienda utilizar la EVRUPP Braden Q (6).

Se logra demostrar que Braden Q es más efectiva para la valoración del riesgo de desarrollar UPP que Norton y concordando con el estudio realizado en el año 2009 (18), se recomienda la utilización de la EVRUPP Braden Q en la población pediátrica.

En un estudio eslovaco la incidencia de úlceras por presión fue del 14\%. La sensibilidad, especificidad, valor predictivo positivo y valor predictivo negativo fueron $85,71 \%, 53,48 \%, 23,07 \%$ y $95,83 \%$, respectivamente, para la escala de Braden (un punto de corte de 15$) ; 85,71 \%, 48,83 \%, 21,42 \%$ y $95,45 \%$, respectivamente, para la Escala de Norton (un punto de corte de 12); y 85,71\%, 30,23\%, 16,66\% y 92,85\%, respectivamente, para la escala de Waterlow (un punto de corte de 13). Las áreas bajo la curva ROC fueron 0,696 (Braden), 0,672 (Norton) y 0,579 (Waterlow). Concluyendo que los mejores valores de validez predictiva, con pequeñas diferencias, se observaron para la escala de Braden, seguido de la Escala de Norton y la escala de Waterlow (19).

Un estudio aleatorizado por grupos no encontró diferencia estadísticamente significativa en la incidencia de úlcera por presión utilizando Braden $(n=74),(R R$ 0,97 IC del 95\%: 0,53 a 1,77) y aquellos pacientes evaluados por opinión de enfermeras mediante la evaluación de riesgos estructurado $(n=106)(\mathrm{RR} 1.43$, IC del 95\%: 0,77 a 2,68), pero un ensayo clínico aleatorizado (ECA) de alta calidad no encontró diferencia estadística en la incidencia de úlceras por presión cuando se evaluaron utilizando cualquiera de las herramienta de evaluación de riesgos, o el uso de juicio clínico. No hay evidencia confiable que el uso de herramientas de evaluación para riesgo de úlcera reduce la incidencia de las úlceras por presión (20).

La escala Braden $\mathrm{Q}$ es la única que muestra unos valores aceptables de validez y capacidad predictiva, aunque su fiabilidad interobservadores no ha sido medida y precisa de más estudios que confirmen estos datos (21).

\section{CONCLUSIONES}

Hasta hoy la escala Braden Q, es un instrumento válido y fiable para valorar el riesgo de desarrollar UPP, y se reduce la sobrevaloración y/o la subvaloración en la que se puede incurrir al realizar una valoración de riesgo de UPP.

Resta investigar tratamientos, rutinas de las unidades de alta complejidad y cómo éstas favorecen o disminuyen el riesgo de desarrollar úlceras por presión; y conocer cómo se comportan estas escalas en unidades de baja complejidad. Se plantea la necesidad de continuar realizando estudios con nuevas escalas, lo que permitiría mayor adherencia a su aplicabilidad.

Es recomendable valorar el riesgo de UPP, aunque con el grado de conocimiento del que hoy disponemos no es posible recomendar ninguna de la escalas de forma 
preferente. Es necesaria más investigación sobre el riesgo de UPP en los niños y sobre la validación de las EVR UPP ya descritas.

Limitaciones del estudio: ayudaría el aumentar el tamaño de muestra, para encontrar relaciones y generalizaciones significativas. Asimismo el acceso a los sujetos de estudio fue otra limitante y el tiempo disponible para investigar el problema y medir el cambio o la estabilidad en el tiempo.

\section{REFERENCIAS BIBLIOGRÁFICAS}

1. Sánchez M, San Sebastián J, Pérez M. Estudio de prevalencia del efecto adverso de úlceras por presión en un hospital de agudos. Enferm Clinic. 2010 [citado 05 Nov 2015]; $20 \quad$ (6):355-359. Disponible en: http://extranet.hospitalcruces.com/doc/adjuntos/Estudio\%20de $\% 20$ prevalencia $\%$ 20de\%20UPP_2010.pdf

2. Kottner J, Wilborn D, Dassen T. Frequency of pressure ulcers in the paediatric population: A literature review and new empirical data. International Journal of Nursing Studies. 2010; [citado 14 Ene 2015]; 47(10):1330-1340 Disponible en: http://www.sciencedirect.com/science/article/pii/S0020748910002178

3. Pancorbo-Hidalgo P, García-Fernández F, Torra i Bou J, Verdú J, Soldevilla-Agreda J. Epidemiología de las úlceras por presión en España en 2013: $4^{\circ}$ Estudio Nacional de Prevalencia. Gerokomos [Internet]. 2014 [citado 13 May 2016]; 25(4): 162-170. Disponible en: http://scielo.isciii.es/scielo.php?script=sci_arttext\&pid=S1134928X2014000400 006

4. Díaz de Durana-Santa S, Ayllon N, Latorre K. Evolución de la incidencia de las úlceras por presión tras la mejora de un protocolo de prevención en cuidados intensivos. Gerokomos [Internet]. 2008 Dic [citado 13 May 2016]; 19 (4): 55-60. Disponible en:

http://scielo.isciii.es/scielo.php?script=sci_arttext\&pid=S1134-928X200800040 0007

5. Drake D, Swanson M, Baker G, Pokorny M, Rose MA, Clark-Reed L, et al. The Association of BMI and Braden Total Score on the Occurrence of Pressure Ulcers, Journal of Wound, Ostomy \& Continence Nursing. 2010 [citado 12 Ene 2015]; 37(4):367-371. Disponible en: http://journals.lww.com/jwocnonline/Abstract/2010/07000/The_Association_of_ BMI_and_Braden_Total_Score_on.5.aspx

6. Noonan C, Quigley S, Curley M. Using the Braden Q Scale to Predict Pressure Ulcer Risk in Pediatric Patients. J Pediatr Nurs.. 2011 [citado 14 Nov 2015]; 26 (6).566-75. http://www.sciencedirect.com/science/article/pii/S0882596310002319

7. Pancorbo P, García F, Soldevilla J, Martínez F. Valoración del riesgo de desarrollar úlceras por presión: uso clínico en España y metaanálisis de la efectividad de las escalas. Gerokomos [revista en Internet]. 2008 Jun [citado 22 Abr 2015]; 19(2): 40-54. Disponible en: http://scielo.isciii.es/scielo.php?pid=S1 134-928X2008000200005\&script=sci_ar ttext 
8. Hospital Hernán Henríquez Aravena. Valoración, Prevención y Notificación de úlceras por presión (UPP). Documentos web. Disponible en: file://E:/Perfil\%202014/Desktop/PUBLI\%20JULIO\%202016/Prevencion_y_not ificacion_de_ulceras_por_presion_upp.pdf

9. García F, Pancorbo P, Soldevilla J. Escalas de valoración del riesgo de desarrollar úlceras por presión en la infancia. Gerokomos [Internet]. 2001 [citado 13 Sep 2015]; 22 (1): 26-34. Disponible en: http://scielo.isciii.es/pdf/geroko/v22n1/helcos1.pdf

10. Curley M, Razmus I, Roberts K, Wypij D. Predicting Pressure Ulcer Risk in Pediatric Patients: The Braden Q Scale. Nursing Research. 2003 [citado 10 Nov 2015]; 52(1):22-33. Disponible en: http://journals.lww.com/nursingresearchonline/Abstract/2003/01000/Predicting _ Pressure_Ulcer_Risk_in_Pediatric.4.aspx

11. Blumel J, Tirado K, Schiele C, Schönffeldt G, Sarrá S. Validez de la Escala de Braden para predecir úlceras por presión en población femenina. Rev Méd de Chile [Internet] 2004 [citado 24 Sep 2015]; 132 (5): 595-600. Disponible en: http://www.scielo.cl/scielo.php?script=sci_arttext\&pid=S0034-98872004000500 009

12. Duffau G. Tamaño muestral en estudios sobre pruebas diagnósticas. Rev. Chil. Pediatr. 1998; 69(3):122-125. [citado 23 May 2015]. Disponible en: http://www.scielo.cl/scielo.php?script=sci_arttext\&pid=S037041061998000300 008

13. Emanuel E. ¿Qué hace que la investigación sea ética? Siete requisitos éticos. OPS/OMS. Serie Publicaciones 1999: Investigación en sujetos humanos: Experiencia Internacional [citado 01 Dic 2015] 33-46. Disponible en: http://www.bioetica.edu.uy/actividades/cbcc6/requisitos_eticos_para_la_investig acion.pdf

14. McLane K, Bookout K, McCord S, McCain J, Jefferson L. The 2003 National Pediatric Pressure Ulcer and Skin Breakdown Prevalence Survey: A Multisite Study. Journal of Wound, Ostomy \& Continence Nursing: 2004 [citado 12 Ene 2015]; 31(4): 168-178 Disponible en: http://journals.lww.com/jwocnonline/Abstract/2004/07000/The_2003_National_ Pediatric_Pressure_Ulcer_and.4.aspx

15. Curley M, Razmus I, Roberts K, Wypij D. Predicting Pressure Ulcer Risk in Pediatric Patients: The Braden Q Scale. Nursing Research [Revista en Internet] 2003 January/February [citado 10 Nov 2015]; 52 (1): 22-33. Disponible en: http://journals.lww.com/nursingresearchonline/Abstract/2003/01000/Predicting Pressure_Ulcer_Risk_in_Pediatric.4.aspx

16. Solis M, Krouskop T, Trainer N, Marburger R. Supine interface pressure in children. Arch Pys Med Rehab. 1988[citado 10 Nov 2015]; 69: 7-12. http://www.ncbi.nlm.nih.gov/pmc/articles/PMC2541103/

17. McCord S, McElvain V, Sachdeva R, Schwartz P, Jefferson L. Risk Factors Associated With Pressure Ulcers in the Pediatric Intensive Care Unit. J Wound Ostomy Continence Nurs. 2004 [citado 14 Ene 2015]; 31 (4): 179-183. http://www.ncbi.nlm.nih.gov/pubmed/15851859

18. Quesada C, Iruretagoyena ML, González RM, Hernández JM, Ruiz de Ocenda MJ, Garitano B et al. Validación de una escala de valoración del riesgo de 
úlceras por presión en niños hospitalizados. Investigación Comisionada. Vitoria-Gasteiz. Departamento de Sanidad y Consumo. Gobierno Vasco, 2009. Informe $\mathrm{n}^{\mathrm{o}}$ : Osteba D-09-08.

19. Šáteková L, Žiaková K, Zeleníková R. Predictive Validity Of The Braden Scale, Norton Scale And Waterlow Scale In Slovak Republic, Cent Eur J Nurs Midw 2015;6 (3):283-290.

20. Moore ZEH, Cowman S. Risk assessment tools for the prevention of pressure ulcers (Review).This is a reprint of a Cochrane review, prepared and maintained by The Cochrane Collaboration and published in The Cochrane Library 2014;2.

21. García F, Pancorbo P, Soldevilla J. Escalas de valoración del riesgo de desarrollar úlceras por presión en la infancia. GEROKOMOS 2015; 22 (1): 26-34. 